\title{
A comparison of intravenous and subcutaneous hydration in elderly acute stroke patients
}

\author{
Yvonne C. Challiner, D. Jarrett, M.J. Hayward ${ }^{2}$, M.A. Al-Jubouri ${ }^{1}$ and \\ S.A. Julious ${ }^{3}$
}

Department of Elderly Services and ${ }^{1}$ Department of Biochemistry, Queen Alexandra Hospital, Cosham, Portsmouth PO6 3 LY, ${ }^{2}$ Medway Hospital, Windmill Road, Gillingham, Kent, and ${ }^{3}$ Department of Medical Statistics and Computing, Southampton General Hospital, Tremona Road, Southampton SO9 4XY, UK

\begin{abstract}
Summary: The aim of this study was to compare the effectiveness of subcutaneous and intravenous fluid therapy in hydrating, elderly acute stroke patients. Thirty-four such patients, needing parenteral fluids because of impaired consciousness or dysphagia, were randomly allocated to receive either subcutaneous or intravenous fluids ( 2 litres of dextrose-saline/ 24 hours). Serum osmolality was measured before starting fluid therapy (Day 1) and on Days 2 and 3. An analysis of covariance of the osmolalities showed no statistical difference between the two groups $(P=0.12)$. The total cost of cannulae used over the 3 days for the subcutaneous route was approximately a third of that for the intravenous route. Complication rates were similar for the two groups. The results suggest that subcutaneous fluid therapy is an effective alternative to the intravenous route.
\end{abstract}

\section{Introduction}

The use of subcutaneous fluid as a method of rehydration is an option sometimes overlooked but worth considering in elderly stroke patients. Such patients often need a period of parenteral hydration because of impaired conscious level and/or dysphagia. Infusion of fluids through the subcutaneous route was first used in paediatric practice in the 1940s. ${ }^{1}$ The method declined in popularity in the 1950s owing to reports of shock caused by osmotic shifts when large amounts of electrolytefree solution were used. ${ }^{2}$

In elderly stroke patients, subcutaneous fluids have a number of potential advantages over intravenous. $^{3-7}$ By sparing the limbs, they offer less hindrance to rehabilitation, and the use of overnight infusions leaves the patient freer to mobilise during the day. As subcutaneous fluids can be started by nursing rather than medical staff, they may allow the more efficient use of human resources. On the other hand, the difficulties and complications of cannulating fragile, elderly veins are well known. ${ }^{4}$

Over the last decade, the safety and effectiveness of subcutaneous fluids in the non-emergency situation have been highlighted and, in our unit, subcutaneous fluids are being increasingly used for the hydration of elderly stroke patients. In a recent study of healthy volunteers, the absorption of

Correspondence: Y.C. Challiner, M.A., M.R.C.P Accepted: 9 September 1993 subcutaneous fluids was found equal to that of intravenous. $^{8}$

The aim of our study was to find out if subcutaneous fluids are effective in restoring hydration in elderly stroke patients when used in routine clinical practice. Serum osmolality was chosen as the biochemical marker of hydration. ${ }^{9}$ We chose to compare subcutaneous fluids with intravenous probably the 'gold standard' of rehydration methods - forming the null hypothesis that there is no difference in the ability of intravenous and subcutaneous fluids to restore biochemical hydration in elderly stroke patients.

\section{Materials and methods}

Thirty-four acute stroke patients admitted consecutively to the Elderly Care Unit and unable to take oral fluids because of impaired conscious level and/or dysphagia, were randomly allocated to receive 2 litres of isotonic dextrose-saline solution (each litre contains $30 \mathrm{mmol}$ of sodium chloride and $40 \mathrm{~g}$ of glucose) per 24 hours via the subcutaneous or the intravenous routes. The study was designed to detect a difference in $10 \mathrm{mOsm} / \mathrm{kg}$ between the two groups with $80 \%$ power and $5 \%$ significance, which required a sample size of 34 . Random treatment allocation was generated by computer, such that within each block of eight patients there were four patients on each treatment. 
These treatment allocations were transferred to sequentially numbered sealed envelopes which were opened by the junior doctor on call once he or she had decided the patient was eligible for the trial and consent had been obtained. The card containing the treatment allocation was then discarded.

Junior doctors and nursing staff had previously been trained in the assessment of swallowing by the Speech and Language Therapy Department, to enable them to assess which patients were suitable for the study. Written consent was obtained from the patient or next of kin.

Venous blood samples were collected into plain 'Vacutainer' tubes for measurement of serum urea, electrolytes, glucose and osmolality, on admission, prior to starting parenteral fluids (Day 1), and on Days 2 and 3 between 9 and 10 a.m. Osmolality was measured using the Osmomat 030 (Clandon, UK). Subjects were kept 'nil by mouth' from admission to the end of Day 3. Exclusion criteria included acute myocardial infarction or any medical condition for which the study fluid regime would be inappropriate. Four patients were excluded for such reasons and two due to lack of consent.

Subcutaneous fluids were delivered through a 19 gauge 'butterfly' cannula sited by a nurse on the trunk, axillary, scapular or thigh areas. The 'butterfly' cannulae were changed every 48 hours. As far as possible, medical and nursing staff ensured the fluids ran to time. Hyaluronidase was not used routinely but if the subcutaneous infusion ran behind time, 1,500 units of hyaluronidase were added to each litre bag of fluid.

Cost of evaluation of total staff time proved impractical, but we did record the numbers, and calculate the cost of the 'Venflon' and butterfly cannulae used. Any complications of the fluid therapy were noted. The study was approved by the Portsmouth and South East Hampshire Health Authority Ethical Committee.

\section{Results}

Seventeen patients received intravenous fluids (i.v.) (age range 71-95, mean age 84.2; 11 female) and 17 received subcutaneous (s.c.) fluids (age range $69-93$, mean age $82.8 ; 11$ female). Not included in the analysis are two patients allocated to the subcutaneous group who dropped out of the study on Day 2: one died and one developed local oedema.

Both treatments reduced plasma osmolality (Figures 1 and 2). On Day 3 the mean osmolality of the i.v. group was below the normal range $(285-295 \mathrm{mOsm} / \mathrm{kg})$ but was within this range in the s.c. group. An analysis of covariance was performed to allow for differences in baselines between the two groups. No statistical difference

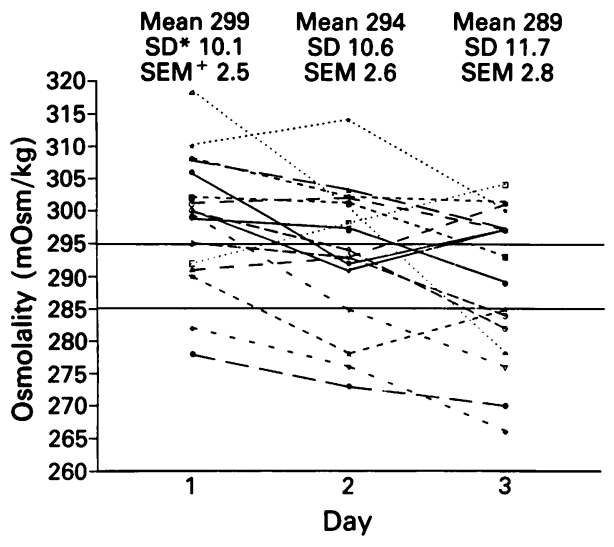

Figure 1 Serum osmolalities of 17 patients receiving subcutaneous fluids Days 1-3 (normal range 285-295 $\mathrm{mOsm} / \mathrm{kg}$ ). *SD denotes standard deviation; + SEM denotes standard error of mean.

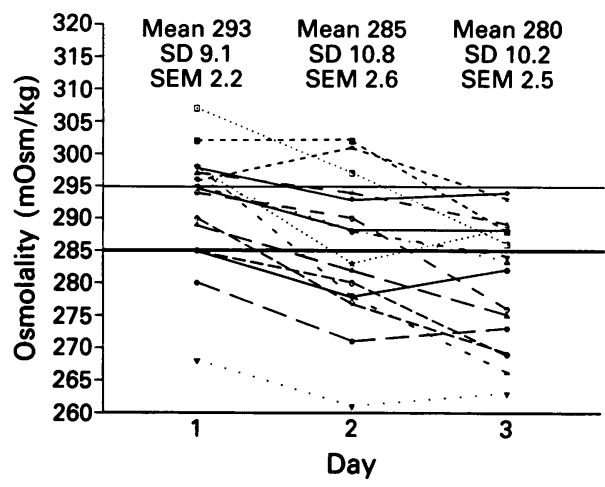

Figure 2 Serum osmolalities of 17 patients receiving intravenous fluids Days 1-3 (normal range 285-295 $\mathrm{mOsm} / \mathrm{kg}$ ).

between the osmolalities of the two treatment groups was found $(P=0.12)$. The least squares estimate of the difference between the i.v. and s.c. groups, given the Day 1 value was estimated to be $5.44 \mathrm{mOsm} / \mathrm{kg}(95 \%$ confidence interval -1.38 to 12.25).

There were two minor local reactions in the s.c. group (erythema) and one in the i.v. (bruising). One patient in the s.c. group required hyaluronidase owing to poor absorption. On Day 3 there were two cases of clinically significant hyponatraemia (taken as serum sodium of 130 or less) in the s.c. group and four in the i.v., although this difference was not statistically significant (Fisher's exact test; $P=$ 0.66). In these instances the fluid regime was stopped or changed as appropriate. 
The total cost of the cannulae used during the three days of the study was $£ 4.93$ for subcutaneous therapy and $£ 13.94$ for intravenous. No problems with venous access were recorded.

\section{Discussion}

This study found no statistical difference in the abilities of subcutaneous and intravenous fluids to restore biochemical hydration in elderly, acute stroke patients. The subcutaneous infusion of fluids has a number of potential advantages over the intravenous route, including simplicity of administration, cost effectiveness and possibly fewer serious complications. We would recommend its consideration as an alternative form of hydration in elderly stroke patients.

The complication rate in each group was minor. Hyponatraemia was the most frequent complication in the intravenous group. A likely contributing factor in both groups was the tendency of stroke to cause syndrome of inappropriate anti-diuretic hormone (SIADH). Factors governing fluid and electrolyte balance in the elderly are complex. ${ }^{10}$ Although for the purposes of this study, all patients

\section{References}

1. Gaisford, W. \& Evans, D.G. Hyaluronidase in paediatric therapy. Lancet 1949, 2: 505.

2. Abbott, W.E., Levey, S., Foreman, R.C. et al. The dangers of administering parenteral fluids by hypodermoclysis. Surgery 1952, 32: 305.

3. Simpson, R.G. Hyaluronidase in geriatric therapy. Practitioner 1977, 219: $361-363$.

4. Schen, R.J. \& Singer-Edelstein, M. Subcutaneous infusion in the elderly. J Am Geriatr Soc 1981, 29: 583-585.

5. Gluck, S.M. \& Rockaway, F. Hypodermoclysis revisited (letter). JAMA 1982, 248: 1310-1311.

6. Schen, R.J. \& Singer-Edelstein, M. Hypodermoclysis (letter). JAMA 1983, 250: 1694

7. Berger, E.Y. Nutrition by hypodermoclysis. J Am Geriatr Soc 1984, 32: 199-203. received the same fluid regime, we would agree with the view of O'Neill et al. that a standard regime may not be appropriate for all patients and that, ideally, frequent biochemical measurements would be used to tailor fluid therapy to individual needs. ${ }^{11}$

Our study looked only at biochemical markers of hydration. Clinical markers of hydration, such as postural hypotension, were not measured and it cannot be assumed that biochemical results would necessarily correlate with clinical impression.

Potentially, subcutaneous fluid therapy could save resources in terms of medical staff time. An analysis of the cost of staff time was beyond the scope of this study. However, we did find significant savings in the cost of the cannulae for subcutaneous therapy. It is possible that the calculated cost of intravenous cannulae used is an underestimate, as junior doctors may not have recorded 'failed attempts'.

\section{Acknowledgements}

We would like to thank all the doctors and nursing staff at Queen Alexandra and St Mary's Hospitals, Portsmouth, for their help and cooperation with this study.

8. Lipschitz, S., Campbell, A.J., Roberts, M.A. et al. Subcutaneous fluid administration in elderly subjects: validation of an under-used technique. J Age-related Disorders 1992, 4: 9-12.

9. McLean, K.A., O'Neill, P.A., Davies, I. \& Morris, J. Influence of age on plasma osmolality: a community study. Age Ageing 1992, 21: 56-60.

10. Miller, M., Gold, G.C. \& Friedlander, D.A. Physiological changes of ageing affecting salt and water balance. Rev Clin Gerontol 1991, 1: 215-230.

11. O'Neill, P.A., Davies, I., Fullerton, K.J. \& Bennett, D. Fluid balance in elderly patients following acute stroke. Age Ageing 1992, 21: $280-285$ 